\title{
List of Reviewers
}

On behalf of ACM Transactions on Modeling and Performance Evaluation of Computing Systems (TOMPECS), we express our thanks to the following people, who have reviewed at least one manuscript for the journal over the past year. We appreciate the time and effort that they put into the reviewing process, which helps our Associate Editors maintain the high-quality standards for the articles that appear in TOMPECS.

$\begin{array}{ll}\text { Mehrnoosh Shafieezade Abade } & \text { Mahmoud Abdelsalam } \\ \text { Varsha Apte } & \text { Islam Mohammad Atiqul } \\ \text { Pariya Babaie } & \text { Gianfranco Balbo } \\ \text { Soumya Basu } & \text { Shajulin Benedict } \\ \text { Simona Bernardi } & \text { Janki Bhimani } \\ \text { Roberto Bifulco } & \text { Alex Borges } \\ \text { Philip N. Brown } & \text { Roberto Bruschi } \\ \text { Yuliya Butkova } & \text { Hector Cancela } \\ \text { Niklas Carlsson } & \text { Laura Carnevali } \\ \text { Davide Cerotti } & \text { Jacob Chakareski } \\ \text { Hyungwon Choi } & \text { Nikolaos Chrysos } \\ \text { Italo Cunha } & \text { Vittoria De Nitto Persone' } \\ \text { Tien Van Do } & \text { Susanna Donatelli } \\ \text { Yajuan Du } & \text { Pierre M. Fiorini } \\ \text { Nicolas Gast } & \text { Javad Ghaderi } \\ \text { Vaibhav Gogte } & \text { Marco Gribaudo } \\ \text { Abhishek Gupta } & \text { Mohammad Hajiesmaili } \\ \text { Nikolas Herbst } & \text { Mingyi Hong } \\ \text { Yaochen Hu } & \text { Yu Hua } \\ \text { Jaehyuk Huh } & \text { Bo Jiang } \\ \text { Xin Jin } & \text { Yanqin Jin } \\ \text { Guillaume Jourjon } & \text { Sang-Woo Jun } \\ \text { Vasileios Karyotis } & \text { Hoda Aghaei Khouzani } \\ \text { William Knottenbelt } & \text { Anne Koziolek } \\ \text { Michael Laurenzano } & \text { Kangwook Lee } \\ \text { Jian Li } & \text { Jun Li } \\ \text { Cong Liu } & \text { Fangming Liu } \\ \text { Ren-Shuo Liu } & \text { Xunyun Liu } \\ \text { Zhenhua Liu } & \text { Catalina Llado } \\ \text { Aniket Mahanti } & \text { Andrea Marin } \\ \text { Daniel Sadoc Menasche } & \text { Ningfang Mi } \\ \text { Maurizio Morisio } & \text { Fabricio Murai } \\ \text { Ryan Nguyen } & \text { Jos Nio-Mora } \\ \text { John Pang } & \text { Marco Paolieri } \\ \text { Brendan Patch } & \text { Naresh M. Patel } \\ & \end{array}$

Alessio Angius
Konstantin Avrachenkov
Siddhartha Banarjee
Daniel S. Berger
Andrea Bianco
Sem Borst
Peter Buchholz
Junwei Cao
Giuliano Casale
Chao Chen
Antonio Cianfrani
Matthias Diener
J. L. Dorsman
Lixin Gao
Anastasios Giovanidis
Zhishan Guo
Xin He
I-Hong Hou
Jiaqing Huang
Yuming Jiang
Gauri Joshi
Weining Kang
Hyoseung Kim
Silvia Krug
Chih-ping Li
Justin Li
Kevin Liu
Yao Liu
Kostas Magoutis
Suzanne Kranjac McIntosh
Christian Moldovan
Giovanni Neglia
Gethin Norman
Alessandro Vittorio Papadopoulos
Massimo Quadrana

(c) 2019 Copyright held by the owner/author(s).

2376-3639/2019/12-ART23

https://doi.org/10.1145/3369841 
Thierry Rakotoarivelo

Charalampos Rotsos

Ahmed Sayed

Olivia Simpson

Joel Sommers

Qihang Sun

Asser N. Tantawi

Max Tschaikowski

Pinghui Wang

Wei Wang

Daniel Wong

Feng Yan

Hui Zang

Hao Zhang

Xiaoxi Zhang

Yangming Zhao
Jia Rao

Marc Sanchez Artigas

Muhammad Shafique

Rachee Singh

Jeremy Sproston

Panagiotis Symeonidis

Gugan Thoppe

Enrico Vicario

Qingyang Wang

Weina Wang

Chuan $\mathrm{Wu}$

Hyunho Yeo

Chaorui Zhang

Hong Zhang

Jian Zhao

Ruiting Zhou
Antonio Augusto Rocha

Matheus Santos

Markus Siegle

Lea Skorin-Kapov

Qifu Sun

Shaojie Tang

Devesh Tiwari

Neil Walton

Sinong Wang

Carey Williamson

Jose Yallouz

Sungjoo Yoo

Fan Zhang

Hongyang Zhang

Ming Zhao

Xingyu Zhou

Sincerely, on behalf of ACM TOMPECS

Sem Borst and Carey Williamson

Co-Editors-in-Chief 